Research Article

\title{
Multioperator Algorithm-Based Ultrasound Imaging Combined with Hysteroscopy for the Diagnosis of Endometrial Polyps
}

\author{
Yousheng $\mathrm{Li} \mathbb{D}^{1}$ and Guitao Xia $\mathbb{D}^{2}$ \\ ${ }^{1}$ Department of Obstetrics and Gynecology, Xinzhou Traditional Chinese Medicine Hospital, Wuhan 430400, China \\ ${ }^{2}$ No. 3 Ultrasound Room, Xinzhou Traditional Chinese Medicine Hospital, Wuhan 430400, China \\ Correspondence should be addressed to Guitao Xia; 2015211124@smail.jsut.edu.cn
}

Received 22 June 2021; Accepted 23 July 2021; Published 4 August 2021

Academic Editor: Gustavo Ramirez

Copyright ( $\odot 2021$ Yousheng Li and Guitao Xia. This is an open access article distributed under the Creative Commons Attribution License, which permits unrestricted use, distribution, and reproduction in any medium, provided the original work is properly cited.

\begin{abstract}
This study aimed to explore the diagnosis of endometrial polyps (EMP) by ultrasound imaging based on multi-operator algorithms combined with hysteroscopy. This study is the first to investigate the resolution of the ultrasound adaptive beamforming algorithm (MOAD), after which the proposed algorithm was applied to ultrasound diagnosis of 102 patients with EMP and pathologically diagnosed with vaginal irregular bleeding, and the evaluation efficacy of the MOAD algorithm based on EMP was compared. The resolution of the MOAD-based imaging algorithm (0.0645) was significantly lower than that of the diagonal loading operator (0.1475), the symbol coherence coefficient operator $(0.1342)$, and the generalized coherence factor operator (0.1234), with significant differences $(P<0.05)$. The proportion of patients with EMP aged 46-55 years was the largest $(55.9 \%)$. There were 64 cases of EMP that produced complications, of which the proportion of patients with uterine fibroids (41.52\%), abnormal uterine bleeding (76.24\%), and menstrual changes $(42.57 \%)$ was relatively large. Patients with nonfunctioning polyps accounted for the largest proportion (84.46\%), followed by those with basal polyps (76.24\%), and the difference was statistically significant $(P<0.05)$. The positive cases of EMP detected by ultrasound imaging $(38$ cases) were significantly lower than those with pathological diagnosis (94 cases), and the difference was statistically significant $(P<0.05)$. The SE, SP, FNR, and FPR of EMP diagnosed by ultrasound imaging combined with hysteroscopy were $64.45 \%, 84.67 \%, 35.48 \%$, and $13.36 \%$, respectively. It has high diagnostic value compared with single ultrasound imaging diagnosis, and the difference was statistically significant $(P<0.05)$. In conclusion, the imaging based on the MOAD algorithm is obvious and the pixel resolution can be successfully improved. The diagnostic value of ultrasound combined with hysteroscopy for EMP was better than that of ultrasound alone $(P<0.05)$, and it had a high diagnostic value.
\end{abstract}

\section{Introduction}

EMP is a very common disease in gynecology, with an incidence rate of $10 \%$ to $35 \%$, and women of reproductive age and elderly women can be affected. It is mainly caused by the excessive sensitivity of some endometrial cells in the endometrium to estrogen, which is a benign lesion of the endometrium [1]. Common symptoms mainly include prolonged menstrual cycle, increased menstrual volume, or nonmenstrual vaginal bleeding, and bleeding is inexhaustible and irregular. It may also be bleeding that is dripping before menstruation and lasts until menstruation, or it may be bleeding that is dripping and bleeding after menstruation, which lasts for ten to twenty days [2]. Most patients have no obvious symptoms, so it is difficult to get timely diagnosis and treatment at an early stage. If EMP is not treated in time, the primary symptoms will gradually worsen, leading to repeated bleeding, foci ulceration, infection, and even infertility. In severe cases, it can even lead to the occurrence of endometriosis, which has a huge impact on the quality of life of patients [3]. Clinically, hysteroscopy is utilized for surgical treatment. For those with longer polyp pedicles, laser 
surgery can be performed under the direct vision of hysteroscopy. However, middle-aged and elderly patients without fertility requirements can have a full or subtotal uterus, so it is imperative to utilize hysteroscopy to diagnose and treat EMP [4].

As an advanced gynecological diagnosis and treatment technology developed in recent years, hysteroscopy can directly show the condition of endometrial lesions, which helps physicians obtain the specific lesion location, size, shape, etc., and improve the diagnostic effect of EMP [5]. However, a single hysteroscopy cannot show the condition of the uterine muscle wall, and there is a phenomenon of missed diagnosis [6]. Medical ultrasound is kind of a medical imaging diagnostic technology based on ultrasound that can visualize the size, structure, and pathological lesions of human muscles and internal organs [7]. In recent years, the clinical performance of vaginal ultrasound has also been quite impressive. It is a noninvasive method that performs rapid continuous scanning and volume data collection. It is also a new method of intuitively obtaining the detailed structure of the uterine cavity, with high sensitivity. Therefore, it was considered that hysteroscopy and vaginal ultrasound could be adopted together to diagnose EMP [8]. Multioperator optimization theory is an optimization idea often utilized in deep learning algorithms. Generally, multiple evolution strategies can be deemed as optimization operators to avoid local convergence when solving singleobjective optimization problems, so as to better find the global optimum solution [9]. Therefore, in this work, it was intended to introduce targeted optimization multioperator to provide ultrasound image quality.

The ultrasonic adaptive beamforming algorithm based on MOAD was proposed, which was compared with multiple operators in terms of evaluation effect on EMP. Then, it was applied in the ultrasonic images of 102 EMP patients, aiming to explore a new clinical diagnosis method for EMP.

\section{Materials and Methods}

2.1. Selection of Research Subjects. From October 30, 2018, to January 10, 2020, 102 patients who were diagnosed as EMP and pathologically diagnosed in the hospital with irregular vaginal bleeding were selected as the research subjects. The age ranged from 25 to 66 years. The study had been approved by the Medical Ethics Committee of Hospital, and the patients and their families understood the situation of the study and signed informed consent forms.

Inclusion criteria: (I) patients without uterine cavity adhesions, submucosal fibroids, and other diseases of the uterine cavity detected by ultrasound; (II) patients who had not received relevant surgical treatment before the test; (III) patients with clear consciousness and able to cooperate with the test.

Exclusion criteria: (I) patients younger than 25 years; (II) patients with mental illness; (III) patients who had undergone surgery; (IV) patients who withdrew from the experiment due to their own reasons.
2.2. Ultrasound and Hysteroscopy Examinations. The ultrasound examination process is as follows. The GE LOGIQ $e$ portable Doppler ultrasound diagnostic instrument (Shanghai Chuxiang Medical Instrument Co., Ltd.) was used to scan patients with an abdominal protrusion probe at a frequency of 5.0 MHz. Before the scan, the patient was asked to empty the bladder and raise the buttocks. The ultrasound probe was smeared with couplers and pushed into the cervical surface for longitudinal, transverse, oblique, and other levels of scanning. The upper and lower diameters, left and right diameters, and anteroposterior diameters of the uterus were recorded. The morphology of the uterus and the echo of the muscle wall were observed, and the endometrial thickness was measured. Diagnostic criteria of EMP are as follows. I: there were single or multiple abnormal echo masses in the uterine cavity, which were basically hyperechoic; II: the two layers of the endometrium were separated and wrapped around the mass, showing the shape of tongue and mulberry; III: the boundary between single polyps and adjacent intima was clear, and the boundary between multiple polyps and normal intima was not uniform and thickened.

The hysteroscopy examination process is as follows. The small hysteroscopy produced by Beijing Uterus Technology Co., Ltd., was adopted for detection. The diameter of the outer sheath was $3.5 \mathrm{~mm}$, the dilatation medium was $6 \%$ glucose solution, and the dilatation pressure was $90 \mathrm{mmHg}$. The bladder biopsy position of the patient was taken during the examination, the cervical canal was expanded, and the hysteroscopy was connected. The cervical canal, intrauterine conditions, and bilateral fallopian tube openings were explored, and the morphology, intimal thickness, and lesion size of the uterine cavity were observed.

\subsection{Ultrasonic Adaptive Beamforming Algorithm Based on} Multioperator Optimization. The ultrasound imaging system can transmit a series of ultrasound beams to the imaging target through the electrical signal of the one-dimensional array probe and then utilize the receiving array element to obtain the echo signals, so as to perform beamforming processing on these signals. Therefore, the basic adaptive beamforming algorithm is first constructed. It is assumed that the number of array elements is $S$; then, the focus echo signal data can be expressed as follows:

$$
e(t)=e\left(t-\frac{\left\|\overrightarrow{e_{t a}}-\overrightarrow{e_{J}}\right\|+\left\|\overrightarrow{e_{e a}}-\overrightarrow{e_{J}}\right\|}{d}\right) .
$$

In equation $(1), \overrightarrow{e_{t a}}$ represents the transmitting array element, $\overrightarrow{e_{J}}$ represents the spatial position of the imaging point, $\overrightarrow{e_{e a}}$ represents the receiving array element, $t$ represents the time of the signal sample, $d$ represents the ultrasonic propagation velocity, and $e$ represents the echo signal data, which can be expressed as follows:

$$
e=z \theta+\alpha+\beta \text {. }
$$


In equation (2), $z$ represents the desired signal of the imaging target, $\theta$ represents the steering vector corresponding to the echo signal, $\alpha$ represents the interference signal vector, and $\beta$ represents the noise signal vector. Then, the beamforming output result can be expressed as follows:

$$
\begin{aligned}
w & =m^{T} e \\
& =\sum_{i=0}^{S-1} m_{\alpha} e_{\alpha} .
\end{aligned}
$$

In equation (3), $m$ represents the weight vector of beam forming and $w$ represents the weighted sum of the output result. The above is the ultrasonic beam forming algorithm. Given that the accuracy of the noise covariance matrix estimation will directly affect the accuracy of the adaptive beamforming algorithm to obtain the apodization weights, firstly, the forward and backward spatial smoothing operator is introduced to obtain the noise covariance matrix as follows:

$$
R=\frac{\sum_{i=1}^{S-H} a_{i} a_{i}^{T}}{S-H+1}
$$

In equation (4), $R$ represents the noise covariance matrix and $a_{i}$ represents the vector of $(H \times 1)$. Then, the obtained $R$ is substituted into equation (3), and the following optimization problem can be obtained:

$$
\begin{gathered}
\underset{m}{\operatorname{Min}} m^{T} R m, \\
\text { subject to } m^{T} \theta=1 .
\end{gathered}
$$

Then, the Lagrange multiplier method can be utilized to calculate the optimal analysis of the problem:

$$
m=\frac{R^{-1} \theta}{\theta^{T} R^{-1} \theta} \text {. }
$$

Such analysis is the apodization weight as beamforming. Given that the frequency deviation of the pulse echo imaging transmitting and receiving signals, the diagonal loading operator is introduced to improve the stability of the covariance matrix. Diagonal loading can superimpose a certain proportion of the identity matrix based on the original algorithm. Then, there is the following equation:

$$
R_{D L}^{*}=R+\lambda * \operatorname{trace}(R) * O .
$$

In equation (7), trace $(R)$ represents the trace of the matrix, $O$ represents the identity matrix, $R *$ represents the matrix after diagonal loading, and $\lambda$ represents the diagonal loading amount, $\lambda \in[(1 / 10 H)-(1 / 100 H)]$. Since the human tissue will affect the ultrasonic propagation speed and make the aperture data unable to be effectively aligned, the sign coherence coefficient operator is proposed to reduce the interference of side lobes and grating lobes, which can be expressed as follows:

$$
F_{\mathrm{SCF}}=\left|1-\sqrt{1-\left(\frac{\sum_{i=1}^{S-1} l_{i}}{S}\right)^{2}}\right|^{k} .
$$

In equation (8), $l_{i}$ represents the value of the sampling point, $l_{i}=\left\{\begin{array}{ll}+1 & z_{i} \geq 0 \\ -1 & z_{i}<0\end{array}\right.$, and $k$ can adjust the sensitivity of the sign coherence factor. Then, the sign coherence factor is taken as the adjustment coefficient operator to adjust the algorithm, and the following equation is acquired:

$$
w_{\mathrm{SCF}}=F_{\mathrm{SCF}} * w \text {. }
$$

Therefore, using the adaptive weighting factor based on phase coherence as the adjustment coefficient of the beamformer output can better suppress side lobes and grating lobes and can improve the final imaging quality. Figure 1 shows the MOAD flow of the ultrasonic adaptive beamforming algorithm optimized by the multiple operator.

The position information of the generalized coherence factor is different from that of the symbol coherence factor, and the interference of the aggregation error can be reduced through the angle transformation. In the calculation, Fourier transform is utilized to transform the gathered various data from the array element domain into the beam domain, and the equation is as follows:

$$
r(l)=e^{j \pi l} \sum_{i=0}^{N-1} T_{i} e^{-j 2 \pi s(l / N)} .
$$

In equation (10), $r(l)$ represents the beam domain function. After the generalized coherence factor is obtained, it needs to be taken as a coefficient operator to adjust the output part of the conventional algorithm. The equation is as follows:

$$
\xi_{\mathrm{GCF}}=a_{\mathrm{GCF}} \cdot \xi .
$$

The apodization weighting algorithm for multioperator adaptive imaging is optimized. The noise covariance matrix can be calculated in the minimum variance beamforming (MV) algorithm. Based on the above theory, the specific optimization equation is as follows:

$$
Z_{\mathrm{MV}+\mathrm{DL}}=\frac{1}{2\left(Z_{\mathrm{MV}}+Z_{\mathrm{DL}}\right)} \text {. }
$$

The noise covariance matrix $Z_{\mathrm{MV}+\mathrm{DL}}$ is optimized, and the following equation is obtained:

$$
V_{\mathrm{MV}+\mathrm{DL}}=\frac{Z_{\mathrm{MV}+\mathrm{DL}}^{-1} \beta}{\beta^{G} Z_{\mathrm{MV}+\mathrm{DL}}^{-1} \beta} \text {. }
$$

In equation (13), $V_{\mathrm{MV}+\mathrm{DL}}$ represents the adaptive apodization weight, and then, the noise is filtered in the signal space to obtain an updated optimized weight: 


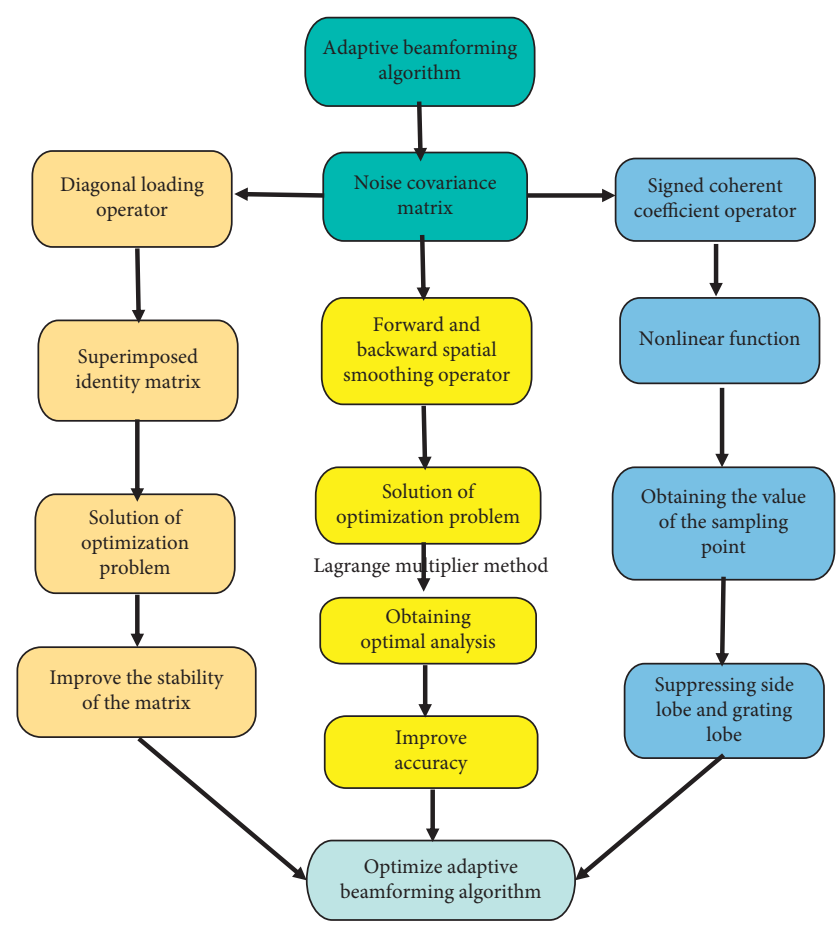

Figure 1: Schematic diagram of the ultrasonic adaptive beamforming algorithm based on multioperator optimization.

$$
V_{\text {MV+DL+update }}=W_{T} W_{T}^{G} V_{\text {MV+DL }}
$$

In equation (14), $W_{T}$ represents the signal subspace finally obtained after the updated apodization weight is eigendecomposed. Using the sign coherence coefficient and the generalized coherence coefficient to optimize the output result in the beamformer can improve the image quality. The calculation equation of the pixel amplitude is as follows:

$$
\varphi_{\mathrm{MV}-\text { range }}=\varphi_{\mathrm{MV}+\mathrm{DL}} \cdot h_{\mathrm{SCF}} \cdot h_{\mathrm{SCF}} \text {. }
$$

After the beamformer outputs the result, the sign coherence coefficient and generalized coherence coefficient adjust its result. Therefore, in the proposed multioperator adaptive imaging algorithm, even if the order of operations is changed, the final imaging effect will not be affected.

2.4. Simulation Parameters. The simulation parameters are as follows. The element spacing was $0.315 \mathrm{~mm}$, the number of array elements was 125 , the number of scanning lines was 125 , the ultrasonic propagation speed was $1500 \mathrm{~m} / \mathrm{s}$, and the focus depth was $25 \mathrm{~mm}$. To evaluate the imaging effect of the multiple-operator algorithm in this research, the application effects of multioperator adaptive ultrasound imaging algorithm and multiple operators were compared through experiments.

The rating index of algorithm imaging quality is mainly the resolution of the region of interest (ROI). In clinical medicine, the resolution of ultrasound imaging is classified into horizontal resolution and vertical resolution. The contrast of the image is usually calculated by the brightness ratio, and the expression is as follows:

$$
\text { Contrast ratio }=\frac{\left(T_{\text {out }}-T_{\text {in }}\right)}{T_{\text {out }}},
$$

where $T_{\text {in }}$ represents the average brightness value in the target area, $T_{\text {out }}$ represents the average brightness value outside the target area, and the value of $T_{\text {out }}$ is the average of the left and right sides, and the calculation is as follows:

$$
T_{\text {out }}=\frac{\left(T_{l}-T_{r}\right)}{2} \text {. }
$$

The Ubuntu 16.0432 bit program was installed to simulate the medical ultrasound imaging scene.

2.5. Statistical Methods. Data were processed by SPSS19.0 version statistical software. Counting data were expressed in frequency and percentage (\%). Statistics of the proportion of age distribution of EMP patients, proportion of fertility, proportion of patients with combined diseases, proportion of clinical symptoms, and pathological classification of endometrial polyps were implemented. The diagnostic value indicators included sensitivity (SE), specificity (SP), false positive rate (FNR), and false negative rate (FPR). The difference was considerable at $P<0.05$.

\section{Results}

3.1. Contrast of Imaging Resolution of Four Algorithms. Figure 2 shows a resolution comparison of the four algorithms. As can be concluded from the figure, the diagonal loading operator had a resolution of 0.1475 ; the resolution of the symbolic coherence coefficient operator was 0.1342 ; the resolution of the generated coherence factor operator was 0.1234; the resolution of the proposed multioperator algorithm was 0.0645 . The resolution of the proposed multioperator algorithm was significantly lower than that of the diagonal loading operator, the signed coherence coefficient operator, and the generalized coherence factor operator, which can successfully improve the pixel resolution, with significant differences $(P<0.05)$.

3.2. Statistics of Information of EMP Patients. Figure 3 shows the age distribution of EMP patients. From the figure, the percentage of EMP patients who were aged 26-35, $36-45,46-55$, and $56-65$ years was $8.8 \%, 23.5 \%, 55.9 \%$, and $11.8 \%$, respectively. Among them, the proportion of patients aged 46-55 years was the largest, indicating that $46-55$ years was the peak of EMP.

Figure 4 was the fertility status of EMP patients. From the figure, the percentages of EMP patients with no history of abortion, one abortion, two abortions, and three or more abortions were $45.15 \%, 30.86 \%, 17.24 \%$, and $6.75 \%$, respectively. It had no history of abortion in 46 patients.

3.3. Ultrasonic Imaging Characteristics of EMP Patients. The ultrasound image of EMP patients showed that the uterine cavity line of the uterus disappeared, and the intrauterine cavity showed enhanced echo, occasionally 


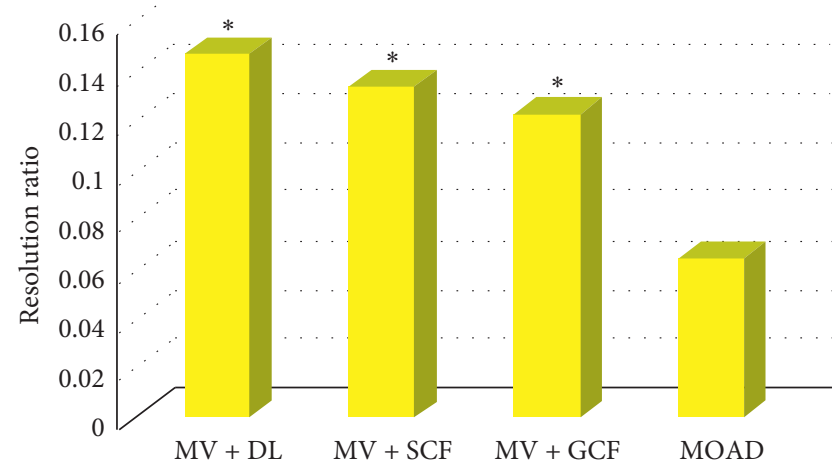

FIgURE 2: Resolution comparison of four algorithms. Note: * represents $P<0.05$ in contrast to the MOAD algorithm.

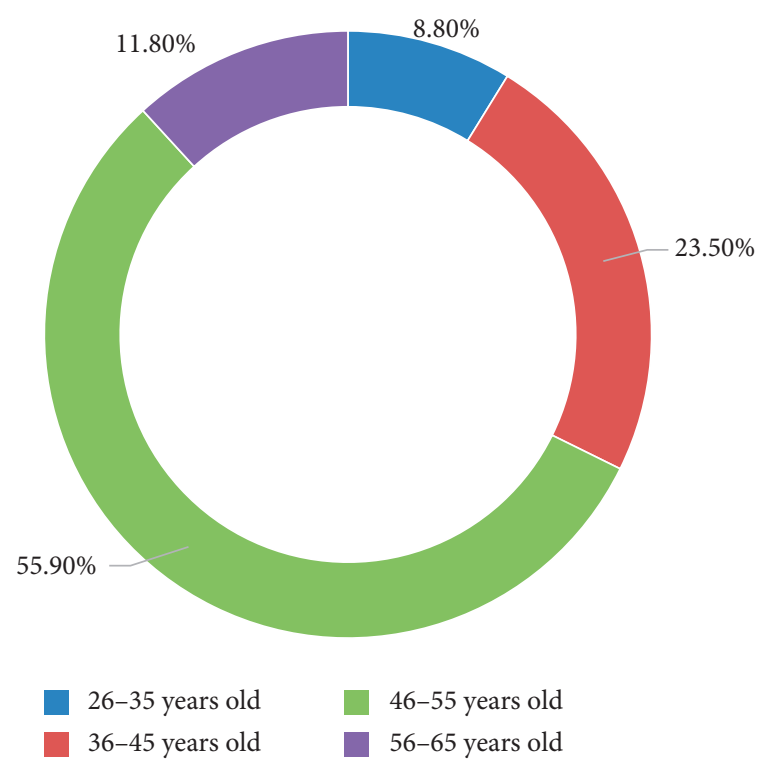

FIgURE 3: Age distribution of EMP patients.

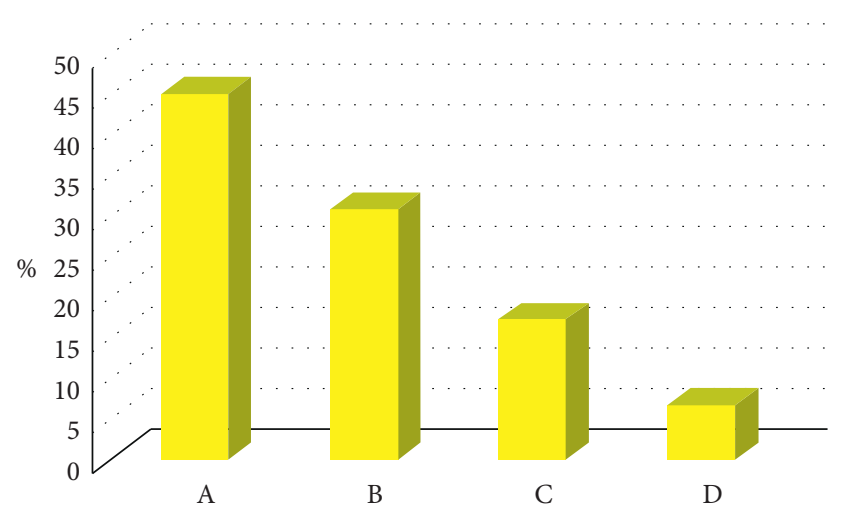

FIGURE 4: Fertility status of EMP patients. (a) No history of abortion. (b) Abortion once. (c) Abortion twice. (d) Abortion three times or more.

uneven hypoechoic. When the middle part of the polyp became cystic, the visible liquid dark area had a clear boundary (Figure 5). The shape of this slightly higher echo

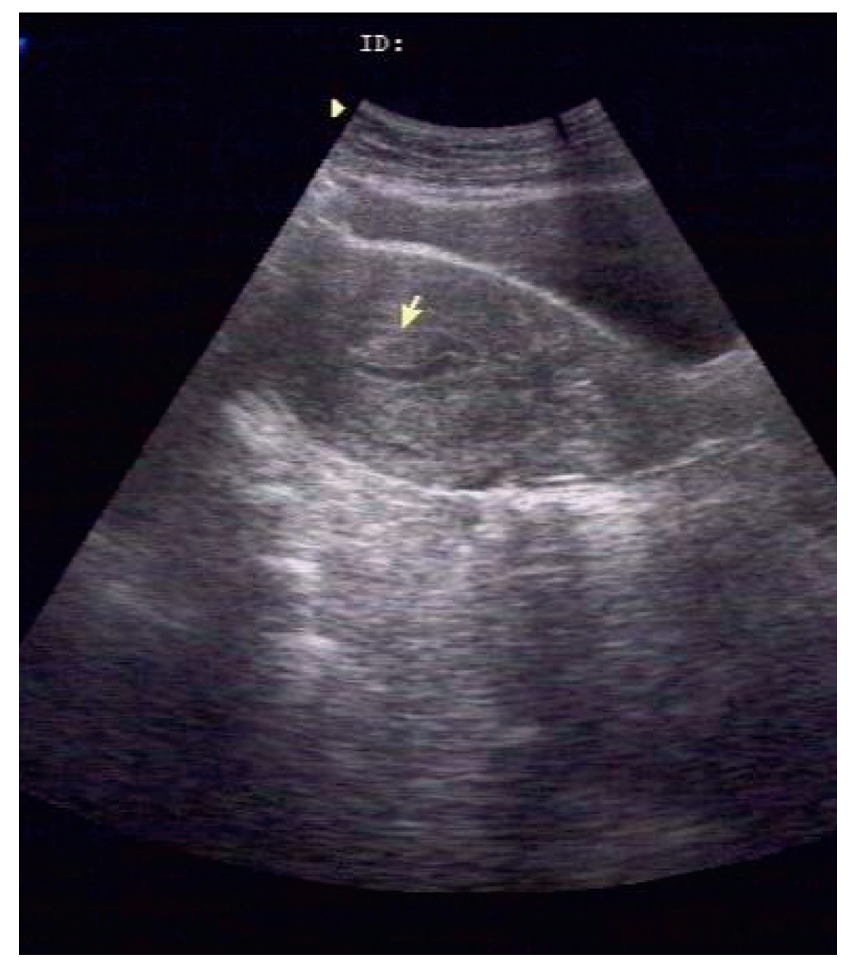

FIgURE 5: Uterine cavity boundary enhancement echo group.

group can be droplet-shaped, band-shaped, round, or oval. The boundary was clear, and blood flow signals can be seen. Polyps can be single or multiple and vary in size, ranging from a few millimeters to a few centimeters. Hyperechoic gas lines can sometimes be seen around the strong echo. It was the more specific manifestation of endometrial polyps, which were completely located in the uterine cavity. Pointlike or short rod-like colored blood flow signals can be seen in the intact muscle of the intimal basement line (Figure 6). Contrast-enhanced ultrasound showed that pedicled or pedicled endometrial masses had a complete endometrial coating.

3.4. Clinical Characteristics of EMP Patients. Figure 7 shows a complication profile in a patient with EMP. From the figure, a total of 64 patients with EMP developed complications. The percentages of EMP patients with uterine fibroids, adenomyosis, and cervical polyps were $41.52 \%$, $8.67 \%$, and $13.48 \%$. The proportion of patients with uterine fibroids was significantly higher than that of patients with adenomyosis and cervical polyps $(P<0.05)$.

Figure 8 shows the clinical symptoms of EMP patients. From the figure, the percentages of EMP patients with abnormal uterine bleeding, menstrual changes, vaginal bleeding, and postmenopausal bleeding were $76.24 \%$, $42.57 \%, 10.58 \%$, and $13.56 \%$, respectively. Among them, the proportion of patients with abnormal uterine bleeding and menstrual changes was significantly higher than that of patients with vaginal bleeding, and the difference was statistically significant $(P<0.05)$. 


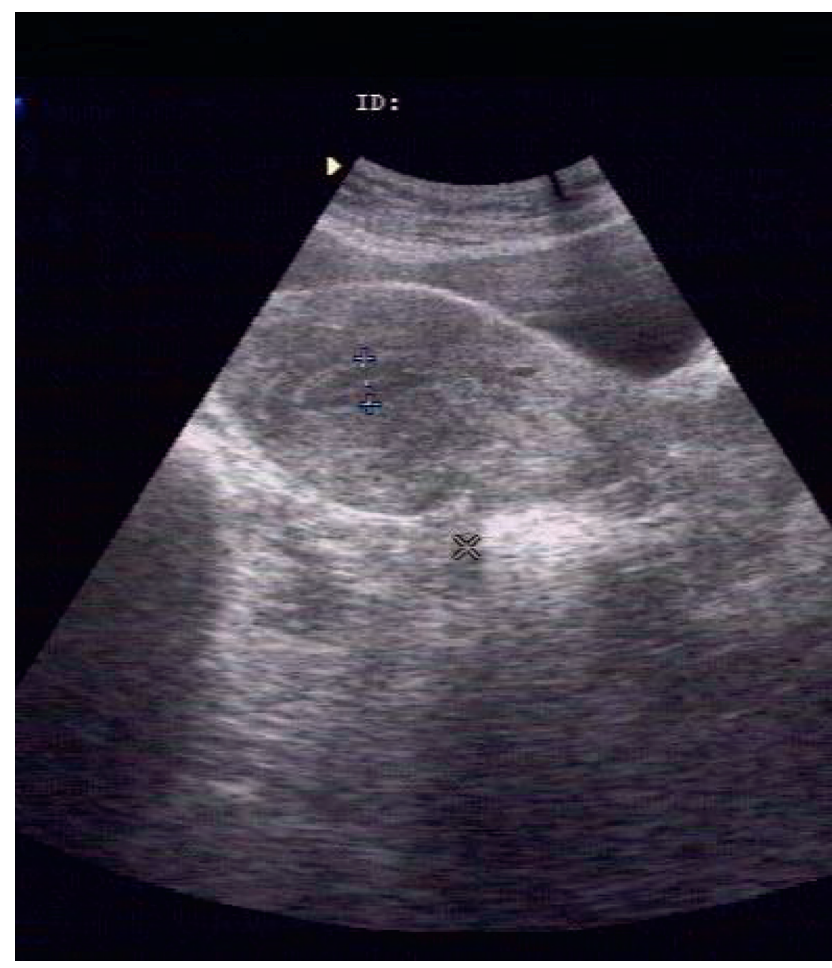

FIgURE 6: Hypoechoic mass in the uterine cavity without vesicles.

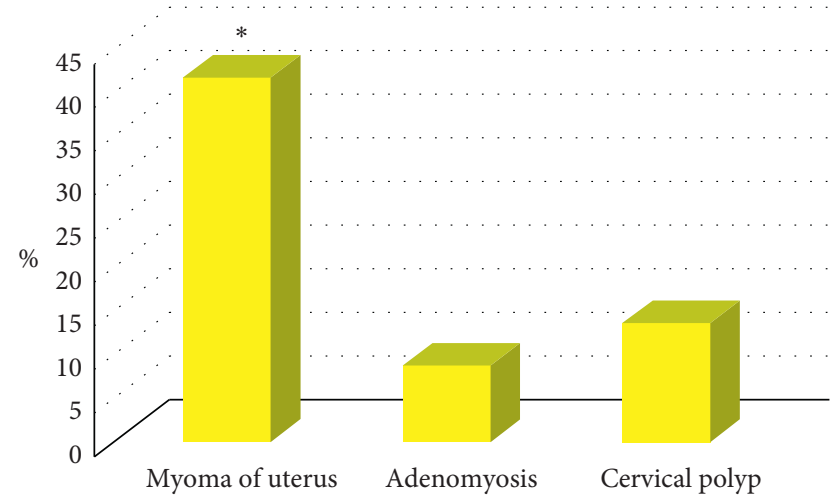

Figure 7: Complications of EMP patients. Note: *indicated $P<0.05$ relative to patients with uterine myopathy and cervical polyps.

\subsection{Pathological Types of Polyps in EMP Patients.} Figure 9 was the polyp type in EMP patients. From the figure, the proportion of patients with functional polyps, nonfunctional polyps, basal polyps, and adenomyoid polyps was $13.5 \%, 84.46 \%, 76.24 \%$, and $4.34 \%$, respectively. Among them, patients with nonfunctioning polyps accounted for the largest proportion, followed by patients with basal polyps, and the difference was statistically significant $(P<0.05)$.

3.6. Contrast of EMP Ultrasound Diagnosis and Pathological Diagnosis. Figure 10 shows the contrast between ultrasound diagnosis and pathological diagnosis results. It was known from the figure that the positive cases of EMP detected by ultrasound imaging (38 cases) were significantly lower than

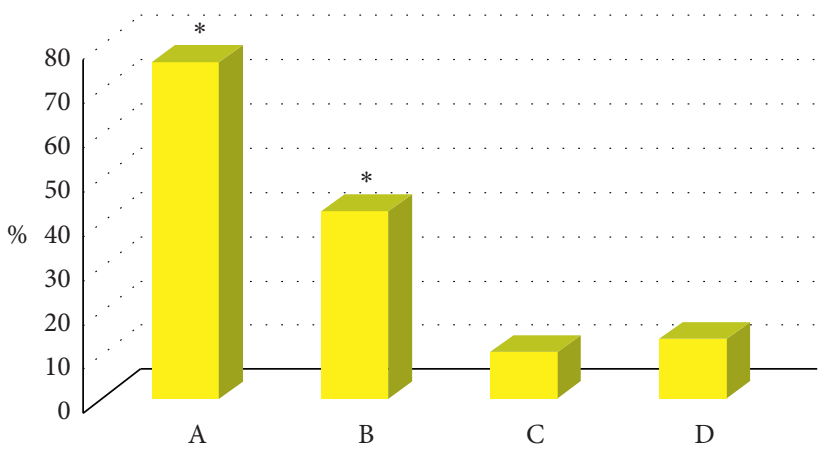

FIgUre 8: Clinical symptoms of EMP patients. (a) Abnormal uterine bleeding. (b) Menstrual changes. (c) Vaginal bleeding. (d) Bleeding after menopause. (*suggested that relative to vaginal bleeding, $P<0.05)$.

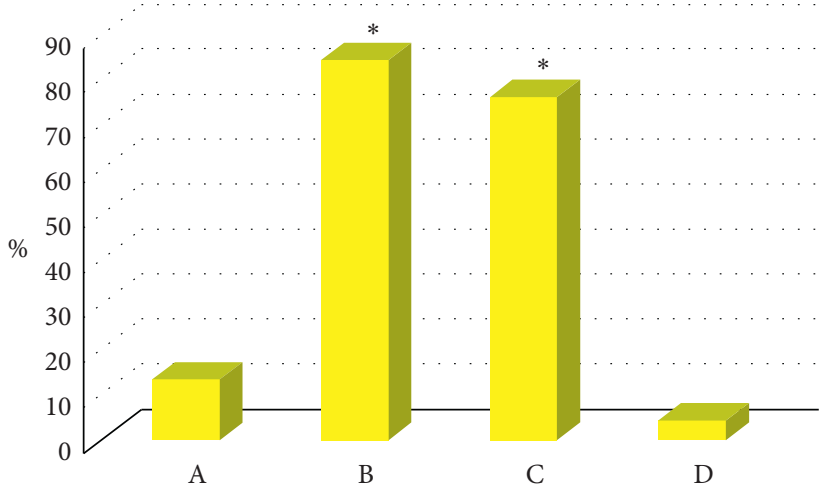

FIGURE 9: Types of polyps in EMP patients. (a) Functional polyp. (b) Nonfunctional polyp. (c) Basal polyp. (d) Adenomyomatous polyp. ( ${ }^{*}$ indicated $P<0.05$ relative to uterine fibroids polyps).

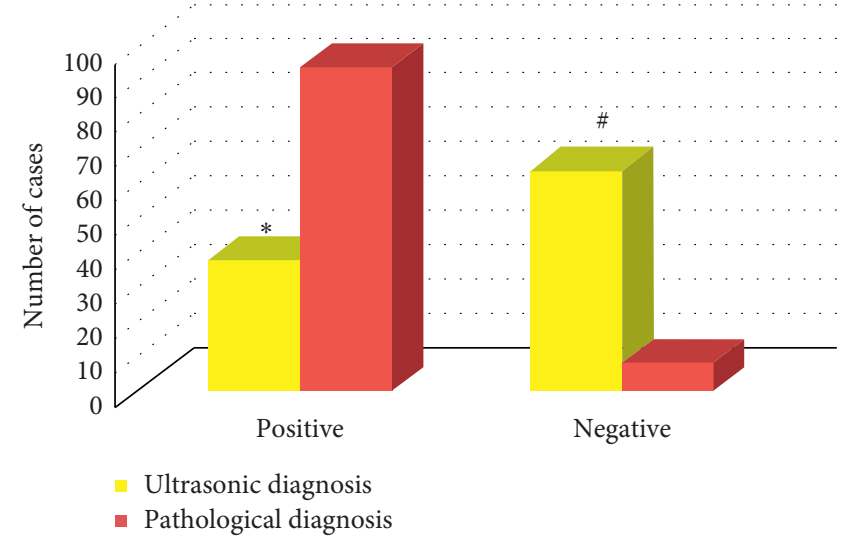

FIgURE 10: Contrast of ultrasound diagnosis and pathological diagnosis results. Note: ${ }^{*}$ indicated $P<0.05$ relative to detected positive cases of EMP by pathological diagnosis, while \# indicated $P<0.05$ relative to detected positive cases of EMP by pathological diagnosis.

those with pathological diagnosis (94 cases), and the difference was statistically significant $(P<0.05)$. The number of EMP negative cases detected by ultrasound imaging (64 
cases) was significantly higher than that of pathological diagnosis ( 8 cases), and the difference was statistically significant $(P<0.05)$.

Figure 11 shows a comparison of the diagnosis results of ultrasound combined with hysteroscopy and pathological diagnosis. As can be concluded from the figure, there is no significant difference between the positive ( 87 cases) and negative (15 cases) cases of EMP patients diagnosed by ultrasound imaging combined with hysteroscopy and the positive (94 cases) and negative (8 cases) cases of clinical pathological diagnosis patients.

\subsection{The Diagnostic Value of Ultrasonic Imaging Combined} with Hysteroscopy for EMP. Figure 12 shows the diagnostic value of ultrasound imaging combined with hysteroscopy for EMP. It can be concluded from the figure that the SE, SP, FNR, and FPR of EMP diagnosed by ultrasound imaging combined with hysteroscopy are $64.45 \%, 84.67 \%, 35.48 \%$, and $13.36 \%$, respectively. Ultrasound imaging combined with hysteroscopy diagnosis had higher diagnostic value than single ultrasound imaging diagnosis, and the difference was statistically significant $(P<0.05)$.

\section{Discussion}

EMP is one of the common diseases in obstetrics and gynecology. Clinical medicine uses conventional ultrasound for diagnosis. The diagnosis method is a noninvasive examination, the operation is simple, and the blood flow of the lesion can be clearly seen $[10,11]$. However, there are reports that ultrasound has a relatively low diagnostic value for endometrial thickness, and hysteroscopy is a minimally invasive examination that requires extremely high accuracy in the examination technology, in order to improve the detection rate of EMP [12]. In this study, the imaging resolution based on the multioperator algorithm was first studied, and the results found that the imaging resolution based on the MOAD algorithm (0.0645) was significantly lower than that of the diagonal loading operator $(0.1475)$ and the symbolic coherence coefficient calculation. The resolution of the sub (0.1342) and the generalized coherence factor operator $(0.1234)$ can successfully improve the pixel resolution, and the difference is very large $(P<0.05)$.

After that, a multioperator-based algorithm was applied to the ultrasound diagnosis of 102 EMPs admitted to our hospital and pathologically diagnosed with vaginal irregular bleeding patients, and the evaluation effects of EMPs were compared between the ultrasound adaptive beamforming algorithm based on multioperator optimization. The results showed that the proportion of patients aged 46-55 years was the largest (55.9\%), indicating that $46-55$ years was the peak of EMP. There were $46(45.15 \%)$ patients who had no history of abortion. This is consistent with van Hanegem et al.'s [13] study, which stated that the peak of EMP was about 40 years old. A total of 64 EMP patients developed complications, and the percentage of patients who developed uterine fibroids (41.52\%) was significantly higher than that of patients who developed adenomyosis (8.67\%) and cervical polyps

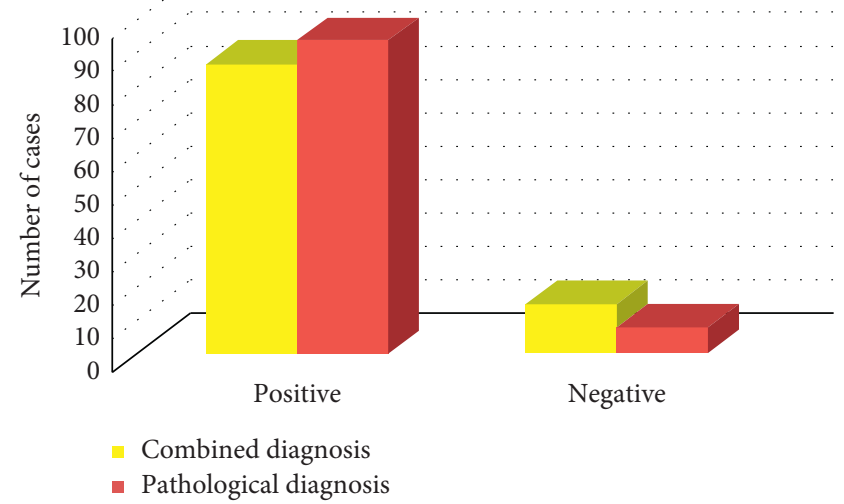

Figure 11: Contrast of diagnosis results of ultrasound combined with hysteroscopy and pathological diagnosis.

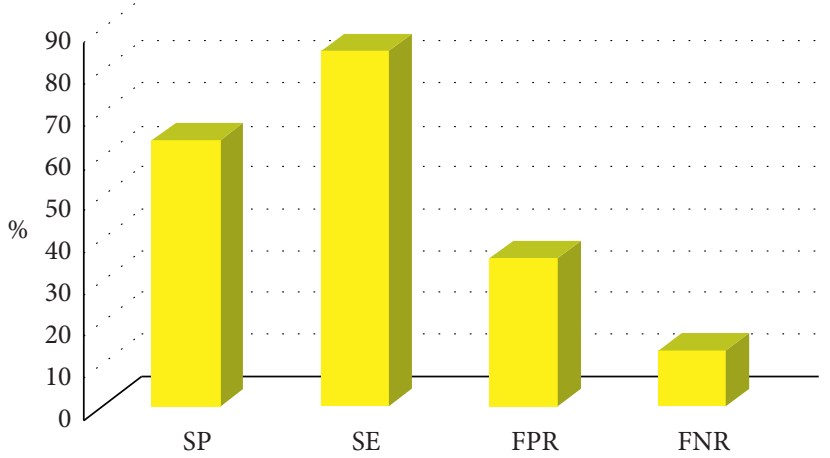

FIGURE 12: The diagnostic value of ultrasonic imaging combined with hysteroscopy for EMP.

(13.48\%) $(P<0.05)$. The proportion of patients with abnormal uterine bleeding (76.24\%) and menstrual changes (42.57\%) was significantly higher than that of patients with vaginal bleeding (13.56\%), and the difference was statistically significant $(P<0.05)$. This is consistent with Bittencourt et al.'s [14] report indicating that about 70\% of EMP patients have abnormal uterine bleeding symptoms, which are most commonly menstrual changes during the parenting phase, such as increased or decreased menstrual volume and variable menstrual timing. In postmenopausal women, vaginal bleeding is the most common. Only a few patients are infertile. Patients with nonfunctioning polyps accounted for the largest proportion (84.46\%), followed by those with basal polyps $(76.24 \%)$, and the difference was statistically significant $(P<0.05)$. The positive cases of EMP detected by ultrasound imaging (38 cases) were significantly lower than those with pathological diagnosis (94 cases), and the difference was statistically significant $(P<0.05)$. The number of EMP negative cases detected by ultrasound imaging (64 cases) was significantly higher than that of pathological diagnosis ( 8 cases), and the difference was statistically significant $(P<0.05)$.

Venturella et al. [15] adopted vaginal ultrasound to diagnose EMP with SE, SP, FNR, and FPR of $45.86 \%$, $96.45 \%, 60.75 \%$, and $1.64 \%$, respectively. The sensitivity and false negative rate of this study were superior to the above 
studies, indicating that vaginal ultrasound had a certain diagnostic value. Vaginal ultrasound combined with hysteroscopy has been widely utilized in the diagnosis of female gynecological diseases in clinical medicine. Since it can clearly observe the situation inside the uterus, it provides great support for clinical diagnosis [16]. The SE, SP, FNR, and FPR of EMP diagnosed by ultrasound imaging combined with hysteroscopy in this study were $64.45 \%, 84.67 \%$, $35.48 \%$, and $13.36 \%$, respectively. Compared with a single ultrasound imaging diagnosis, it had a higher diagnostic value, and the difference was statistically significant $(P<0.05)$. Banas et al. [17] pointed out that the SE, SP, FNR, and FPR of EMP diagnosed by vaginal ultrasound combined with hysteroscopy were $97.45 \%, 93.26 \%, 3.89 \%$, and $4.37 \%$, respectively. Relative to the evaluation of ultrasonic imaging, the diagnostic value of ultrasonic imaging combined with hysteroscopy was higher $(P<0.05)$.

\section{Conclusion}

In this study, the resolution imaged by the multioperator based algorithm was firstly investigated, after which the evaluation effects of the proposed ultrasound adaptive beamforming algorithm based on multioperator optimization and the ultrasound adaptive beamforming algorithm based on multioperator optimization on EMP were compared. The results showed that the imaging effect of the target range was obvious, and the pixel resolution can be successfully improved. The peak period of onset of EMP patients was 46-55 years old, and the main clinical symptom of EMP was abnormal uterine bleeding. The pathological types of EMP were mainly nonfunctional endometrial polyps, among which basal endometrial polyps were the most common. Vaginal ultrasound had a certain diagnostic value in EMP, and ultrasonic imaging combined with hysteroscopy had a higher diagnostic value, which can evidently reduce the occurrence of missed diagnosis. The shortcoming of this study is that the selected sample size is small, which leads to biased results. Later, it will consider expanding the sample range for further research. In short, this study provides a reliable diagnostic method for the clinical diagnosis of EMP.

\section{Data Availability}

The data used to support the findings of this study are available from the corresponding author upon request.

\section{Conflicts of Interest}

The authors declare that they have no conflicts of interest.

\section{References}

[1] F. Gu, H. Zhang, S. Ruan et al., "High number of endometrial polyps is a strong predictor of recurrence: findings of a prospective cohort study in reproductive-age women," Fertility and Sterility, vol. 109, no. 3, pp. 493-500, 2018.

[2] Y. Gui, L. Wang, T. Gao, H. Hong, and W. Zhao, "Ultrasonic imaging combined with hysteroscopy in diagnosis of endometrial polyps based on multioperator algorithm and analysis of nerve growth factor receptor t protein expression," World Neurosurgery, vol. 149, no. 20, pp. 413-419, 2021.

[3] A. Kumari, S. Pankaj, V. Choudhary et al., "Ultrasonic and histopathological evaluation to exclude premalignant and malignant lesions in perimenopausal and postmenopausal women presenting as abnormal uterine bleeding," Journal of Obstetrics \& Gynaecology of India, vol. 69, pp. 171-176, 2019.

[4] T. J. Clark and H. Stevenson, "Endometrial Polyps and Abnormal Uterine Bleeding (AUB-P): what is the relationship, how are they diagnosed and how are they treated?" Best Practice \& Research Clinical Obstetrics \& Gynaecology, vol. 40, pp. 89-104, 2017.

[5] Y. Chen, S. Hu, H. Mao, W. Deng, and X. Gao, "Application of the best evacuation model of deep learning in the design of public structures," Image and Vision Computing, vol. 102, Article ID 103975, 2020.

[6] L. Adomaitienė, R. Nadišauskienè, M. Nickkho-Amiry et al., "Proliferation in postmenopausal endometrial polyps-A potential for malignant transformation," Medicina, vol. 55, no. 9, p. 543, 2019.

[7] L. M. P. Sasaki, K. R. C. Andrade, A. C. M. G. Figueiredo, M. d. S. Wanderley, and M. G. Pereira, "Factors associated with malignancy in hysteroscopically resected endometrial polyps: a systematic review and meta-analysis," Journal of Minimally Invasive Gynecology, vol. 25, no. 5, pp. 777-785, 2018.

[8] Y. Sun, J. Zhang, and W. Bai, "Higher prevalence of endometrial polyps in patients with fallopian tube obstruction: a case-control study," Journal of Minimally Invasive Gynecology, vol. 26, no. 5, pp. 935-940, 2019.

[9] Z. Lv and W. Xiu, "Interaction of edge-cloud computing based on SDN and NFV for next generation IoT," IEEE Internet of Things Journal, vol. 7, no. 7, pp. 5706-5712, 2020.

[10] A. Ghoubara, S. Sundar, and A. A. A. Ewies, "Predictors of malignancy in endometrial polyps: study of 421 women with postmenopausal bleeding," Climacteric, vol. 21, no. 1 , pp. 82-87, 2018.

[11] Y. A. Tohma, G. Onalan, S. Esin et al., "Are there any predictors of endometrial premalignancy/malignancy within endometrial polyps in infertile patients?" Gynecologic and Obstetric Investigation, vol. 84, no. 5, pp. 512-518, 2019.

[12] H. Zhang, X. He, W. Tian, X. Song, and H. Zhang, "Hysteroscopic resection of endometrial polyps and assisted reproductive technology pregnancy outcomes compared with No treatment: a systematic review," Journal of Minimally Invasive Gynecology, vol. 26, no. 4, pp. 618-627, 2019.

[13] N. van Hanegem, M. M. C. Prins, M. Y. Bongers et al., "The accuracy of endometrial sampling in women with postmenopausal bleeding: a systematic review and meta-analysis," European Journal of Obstetrics \& Gynecology and Reproductive Biology, vol. 197, pp. 147-155, 2016.

[14] C. A. Bittencourt, R. dos Santos Simões, W. M. Bernardo et al., "Accuracy of saline contrast sonohysterography in detection of endometrial polyps and submucosal leiomyomas in women of reproductive age with abnormal uterine bleeding: systematic review and meta-analysis," Ultrasound in Obstetrics and Gynecology, vol. 50, no. 1, pp. 32-39, 2017.

[15] R. Venturella, G. Miele, K. Cefalì et al., "Subcutaneous progesterone for endometrial polyps in premenopausal women: a preliminary retrospective analysis," Journal of Minimally Invasive Gynecology, vol. 26, no. 1, pp. 143-147, 2019.

[16] T. Kınay, Z. Öztürk Başarır, S. Fırtına Tuncer, F. Akpınar, F. Kayıkçığlu, and S. Koç, "Prevalence of endometrial polyps 
coexisting with uterine fibroids and associated factors," Journal of Turkish Society of Obstetric and Gynecology, vol. 13, no. 1, pp. 31-36, 2016.

[17] T. Banas, K. Pitynski, M. Mikos, and J. Cielecka-Kuszyk, "Endometrial polyps and benign endometrial hyperplasia have increased prevalence of DNA fragmentation factors 40 and 45 (DFF40 and DFF45) together with the antiapoptotic B-cell lymphoma (Bcl-2) protein compared with normal human endometria," International Journal of Gynecological Pathology, vol. 37, no. 5, pp. 431-440, 2018. 